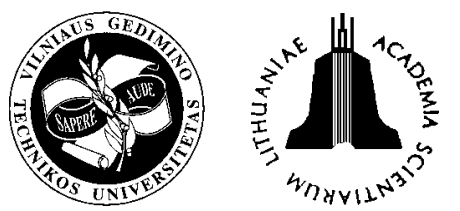

\title{
SIMULATION MODELING OF THE GROUP OF MULTIMODAL CARGO LINES INCLUDING WATER TRANSPORT
}

\author{
Nikolay Kazakov \\ Department of Exploitation Work Management, \\ Belarusian State University of Transport, Kirov str. 34, 246653 Gomel, Belarus, \\ Phone: +375 232952 184, E-mail: koliandr@tut.by \\ Received 12 January 2006, accepted 28 March 2006
}

\begin{abstract}
The aim of this paper is to present the concept of making a simulation model of the multimodal cargo lines group including water transport and the principles of its formation in the form of interconnected similar modules. The paper presents a generalized algorithm for choosing the optimum multimodal transportation variant on the basis of simulation modeling. The matter of the paper is given on the example of the organization of export and import cargoes multimodal transportation by Belarus in southern direction.
\end{abstract}

Keywords: multimodal transportation, multimodal cargo line, simulation model, transport fleet.

\section{Introduction}

Nowadays the tendencies of world market have been formed thus that the majority of goods owners have to refuse the traditional system of goods delivery, when each mode of transport is used separately from the other and to use integrated multimodal transportation. The system of measures for organizing the transportation of cargoes and the motion of mobile units according to the system of multimodal cargo lines is an important reserve of the logistic effectiveness of goods delivery from the owner to the consumer. The purpose of the research is to reveal the conditions maximizing the value of this effect.

The multimodal cargo lines which are grouped according to the definite indication (for example, according to the fleet which is used for goods transportation or according to goods traffics) and have their specific character and indices are the object being researched.

As a result of the development of the queuing theory methods and the integration of the computer technology in science and production, the methods of simulation modeling for the complex systems searching became widespread. Therefore, this method is selected as the method to research the effectiveness of the functioning organization of the transport taking part in the multimodal transportation.

\section{The definition of the multimodal cargo line}

Though multimodal and intermodal transportations are widely used in the world transportation practice, until now different interpretation of the mixed transportation terminology is given in the contemporary literature [1]. That is why the terms used in this article require explanation and strict definition.

We will name the transportation of cargos by several modes of transport "multimodal" when one transporter or operator takes responsibility for the entire transportation of the cargo from the supplier to the consumer. In this case, a single transport document is used to perform the cargo transportation.

The essence of the term "multimodal cargo line" can be revealed on the grounds of the syntheses of the notion "multimodal transportation" (described above) and the notion "cargo line", which is used in the water transport exploitation science, by which the transport connection between the definite points of similar types of cargo departure and destination is regarded regularly performed by single-type fleet during the entire period of cargo transportation [2]. Under cargo "multimodal" line the system is understood organized by the operator of the transport service of both-way cargo traffics between definite points of loading and unloading performed by two or more modes of transport using a single transport document. The departure of transport units is regulated 
by definite departure intervals (frequencies) and their motion is regulated by a graph or by a timetable.

Taking into consideration that the term "multimodal cargo line" is a wider notion than the term "cargo line", it is necessary to note that the condition of transport units departure is regulated by a graph or by a timetable and implies the functioning of water transport according to the linear form of the motion organization, i.e., the functioning with the use of the fixed group of one-type vessels, as the change of kind or type of transportation fleet significantly changes the whole transport process technology. However, such a rigid fixing during the multimodal cargo line functioning is not obligatory concerning the use of rolling stock of the other modes of transport, though, in this case the regulation of a transport process must be fulfilled anyway.

\section{Motivation of the research method choice}

A continuous time varying complex dynamic process occurs in any transport system. That is to say the system being researched assumes the specific state at each fixed moment of time. Consequently, the process of this system functioning can be considered as the sequential change of its states. Herewith, the functioning of such a complex dynamic system as a multimodal cargo line represents the combination of the sequential and parallel elementary operations fulfillment.

All the methods used to study the technological aspects of water-transport systems functioning, can be divided into three groups:

1) methods based on empirical dependence;

2) methods of queueing theory;

3) methods of simulation modeling.

The methods of the system researching based on empirical dependences were quite extensively used before the appearance of an analytical system for the description of stochastic processes. Empirical dependences obtained for the specific conditions have narrow, limited by the given conditions application.

The analytical dependences of the queueing theory for the definition of the systems functioning characteristics are based on the comparatively simple cases (Poisson distribution of the incoming flow, single, uniform requests and etc.), consequently these dependences at relatively low detailing of the specific system properties researching are rather approximate.

A precise experiment, the construction of physical analogs when modeling the systems, transport ones as well, are connected with high expenditures of time and resources, and are often associated with significant organizational difficulties. In such cases, as the research method of different aspects of the complex system functioning, one should use the methods of simulation modeling, which in connection with the computer technology development have received wide acceptance in scientific researches and in the solution of practical problems [3, 4].

\section{Model of a multimodal cargo line group}

The principles of universal simulation models construction are often used for the purposes of algorithmic unification of transport systems modeling [3, 4]. The main principle of such models construction is mathematical uniform description of all the elements of the system being researched in the form of an elementary model i.e. module.

The diagrammatic representation of the $k$-th order module is represented in Fig 1.



Fig 1. Module describing the elementary operation: $I^{k}$ is the vector of the incoming flow; $f r^{k}$ is the vector of the outgoing flow $n_{w t}^{k}$ is the post of service-time expectance; $n_{w t}^{k}$ is the post of the request servicing; $S^{k}$ is the module state vector

The process of the $j$-th order module functioning is the sequential change of the module states, which is described by the expression:

$$
M^{j}=\left|\begin{array}{lll}
S^{j-1} & \stackrel{A_{c h}^{j}}{\longrightarrow} & S^{j} \\
I^{j} & \stackrel{A_{\text {transf }}^{j}}{\longrightarrow} & f r^{j}
\end{array}\right| .
$$

As a result of the loads on module $I^{j}$, by means of the module state transition algorithm $A_{c h}^{j}$ the new module state vector $S^{j}$ is worked out, while by means of the transformation algorithm $A_{\text {transf }}^{j}$ of the loads on the module $I^{j}$ the vector of the outgoing low $\mathrm{fr}^{k}$ is worked out into the outgoing flow of the module. That is to say, the $j$-th order module can be unambiguously defined by the totality of the vectors $I^{j}, f r^{k}, S^{j}$, of the algorithms $A_{c h}^{j}, A_{\text {transf }}^{j}$, of the tasks solved by the module and of the multitude of the modeling time moments $t$ :

$$
M^{j}=\left(t, b, I^{j}, f r^{j}, S^{j}, A_{c h}^{j}, A_{\text {transf }}^{j}\right) .
$$

Consequently, multitudes are mathematical models of the system which is used for formal description of the modules conjunction:

$$
\bigcup_{i=1}^{n_{\text {ent }}} I, \bigcup_{i=1}^{n_{\text {imp }}} f r, \bigcup_{i=1}^{n_{\text {cond }}} S, \bigcup_{i=1}^{n_{\text {ch }}} A_{c h}^{j}, \bigcup_{i=1}^{n_{\text {transf }}} A_{\text {transf }}^{j}
$$


where: $n_{\text {ent }}$ and $n_{\text {imp }}$ are accordingly a quantity of input and output system flows; $n_{\text {cond }}$ is a quantity of probable system states; $n_{c h}$ is a quantity of the state transmission algorithms; $n_{\text {transf }}$ is a quantity of the algorithms of the incoming flows into outgoing flows transformation.

The process of multimodal cargo line functioning is formalized on the basis of the modular principle of the simulation models description. The model of simulation models (module of the $1^{\text {st }}$ order) combines:

- modules that adequately describe simple technological operations (documenting, setting the vessel in the roadstead, trans-shipment from the wagons onto the vessels, shunting operations with wagons etc.) are the modules of the $3^{\text {rd }}$ order;

- modules, that characterize the operations of the transportation process (railway rolling stock servicing at the technical stations, the motion of fleet at sections etc.) are the modules of the $2^{\text {nd }}$ order.

For the visual characteristic of the module, one should use the following conditional symbolism proposed in the source consisting of four parameters: the first parameter characterizes the flow of requirements $(P-$ the flow of requirements can have a different law of entering requests distribution); the second parameter characterizes the random sequences of the request servicing duration ( $F-$ the request servicing can have varying duration); the third parameter determines a quantity of operating channels; the fourth parameter determines a service discipline (if the fourth parameter is omitted, the priority of the request servicing is absent).

When forming the simulation model of the multimodal cargo lines group, simple operations can be adequately described in the form of three types of modules: $P / P / 1, P / P / 1 / F, P / P / n / F$ and $P / P / n$.

Physical sense of the modules of the type $P / P / 1$ and $P / P / n$ lies in the following: the flow of the requests $I^{j}$ enters into the module at random time intervals $t$. The law of distribution of time intervals between the requests enterings can be different. Requests are queued and serviced according to the entering. Module has one (module $P / P / 1$ ) or $n$ $(P / P / n)$ channels of servicing. The duration of servicing is a random variable. In the system being researched the means of mechanization ( $n$ of units), which maintains the transportation means, a locomotive (or a group of locomotives) working accumulating the wagons etc. are examples of the modules of the type $P / P / n$.

With the presence of priority servicing of the requests, the modules characterizing such subsystems are designated by $P / P / 1 / F$ or $P / P / n / F$, depend- ing on the number of servicing channels. This module gets $P / P / n / F$ of the incoming requests flows, each of them is subordinated to its distribution law. Requests are unequal by the importance. Laws of distribution of the requests income time intervals as well as of its servicing duration can be different.

Mathematically the modules of the $j$-th order can be recorded in the following form:

1) with parallel functioning of modules of the $(j+1)$-th order for solving of the $b$-th task:

$$
M_{1}^{j}=\left|\begin{array}{c}
M_{1}^{j+1} \\
M_{2}^{j+1} \\
\vdots \\
M_{m}^{j+1}
\end{array}\right|=\max _{t}\left\{M_{i}^{j+1}\right\},
$$

where: $t$ is a parameter characterizing the process of module functioning, the time stands as such parameter for the models of transport system and of the overwhelming majority of the other complex systems;

2) with the sequential functioning of $m$ modules of the $(j+1)$-th order for solving of the $b$-th task:

$$
M^{j}=\left|M_{1}^{j+1} M_{2}^{j+1} M_{3}^{j+1} \ldots M_{m}^{j+1}\right|=\sum_{t} M_{i}^{j+1} ;
$$

3) combining the parallel and sequential modules functioning:

$$
\begin{aligned}
& M_{1}^{j}=\left|\begin{array}{c}
M_{1}^{j+1} \\
M_{2}^{j+1} \\
\vdots \\
M_{m}^{j+1}
\end{array}\right|=\left|\begin{array}{lll}
M_{m+1}^{j+1} & \ldots & M_{m+k}^{j+1}
\end{array}\right|= \\
& \max _{t}\left(\max _{t}\left\{M_{i}^{j+1}\right\} ; \sum_{t} M_{m+i}^{j+1}\right) .
\end{aligned}
$$

Since the moment of computer technology wide application the methods of simulation modeling have been repeatedly used to research complex systems, including transport systems [4], therefore algorithms of different types elementary modules functioning, including $P / P / 1, P / P / 1 / F, P / P / n / F$ and $P / P / n$ recommended themselves well and can be practiced without changes to form the model of the multimodal cargo lines group.

On the basis of the designations described above and technology of transport objects functioning let's consider the examples of the records of the modules of cargos delivery operations in multimodal communication. The interpretation of the matter is constructed in connection with the functioning of the multimodal export and import cargo lines from 
Belarus to the Ukrainian Black sea ports and the ports of the Danube Basin countries. The scheme of waterway is represented in Fig 2.

Railway rolling stock servicing at the technical stations of the Belarusian railway:

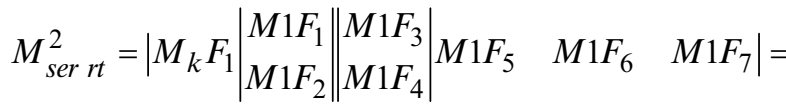

$$
\begin{aligned}
& M_{k} F_{1}+\max \left(M 1 F_{1} ; M 1 F_{2}\right)+\max \left(M 1 F_{3} ; M 1 F_{4}\right)+ \\
& \sum_{m=5}^{7} M 1 F_{m}
\end{aligned}
$$

where: $M_{k} F_{1}$ is a module of cargo operations with wagons ( $k$ is a quantity of channels performing cargo operations, which is determined by a technological process); $M 1 F_{1}$ is wagons accumulation in the train; $M 1 F_{2}$ is the documenting in the transport servicing control center; $M 1 F_{3}$ is commercial inspection of the train; $M 1 F_{4}$ is technical inspection of the train; $M 1 F_{5}$ is train preparation for the departure; $M 1 F_{6}$ is the expectation of the road schedule; $M 1 F_{7}$ is the train departure.

Automobile road-train servicing:

$$
\begin{aligned}
& M_{\text {ser at }}^{2}=\left|M_{k} F_{1}\right| \begin{array}{c}
M 1_{1} \\
M 1 F_{2}
\end{array}\left|M 1 F_{3}\right|= \\
& M_{k} F_{1}+M 1 F_{1}+\max \left(M 1_{1} ; M 1 F_{2}\right)+M 1 F_{3},
\end{aligned}
$$

where: $M_{k} F_{1}$ is a module of technical inspection and servicing of the automobile ( $k$ is the quantity of service channels); $M 1 F_{1}$ is the displacement of the automobile to the warehouse of the cargo owner; $M 1_{1}$ is the documenting; $M 1 F_{2}$ is cargo loading into the automobile; $M 1 F_{3}$ is commercial inspection of the cargo.

Belarusian fleet servicing in the ports of Belarus:

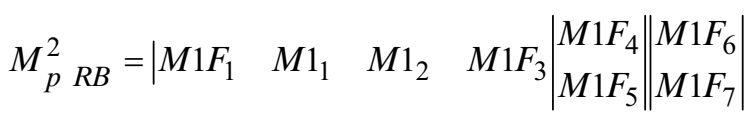

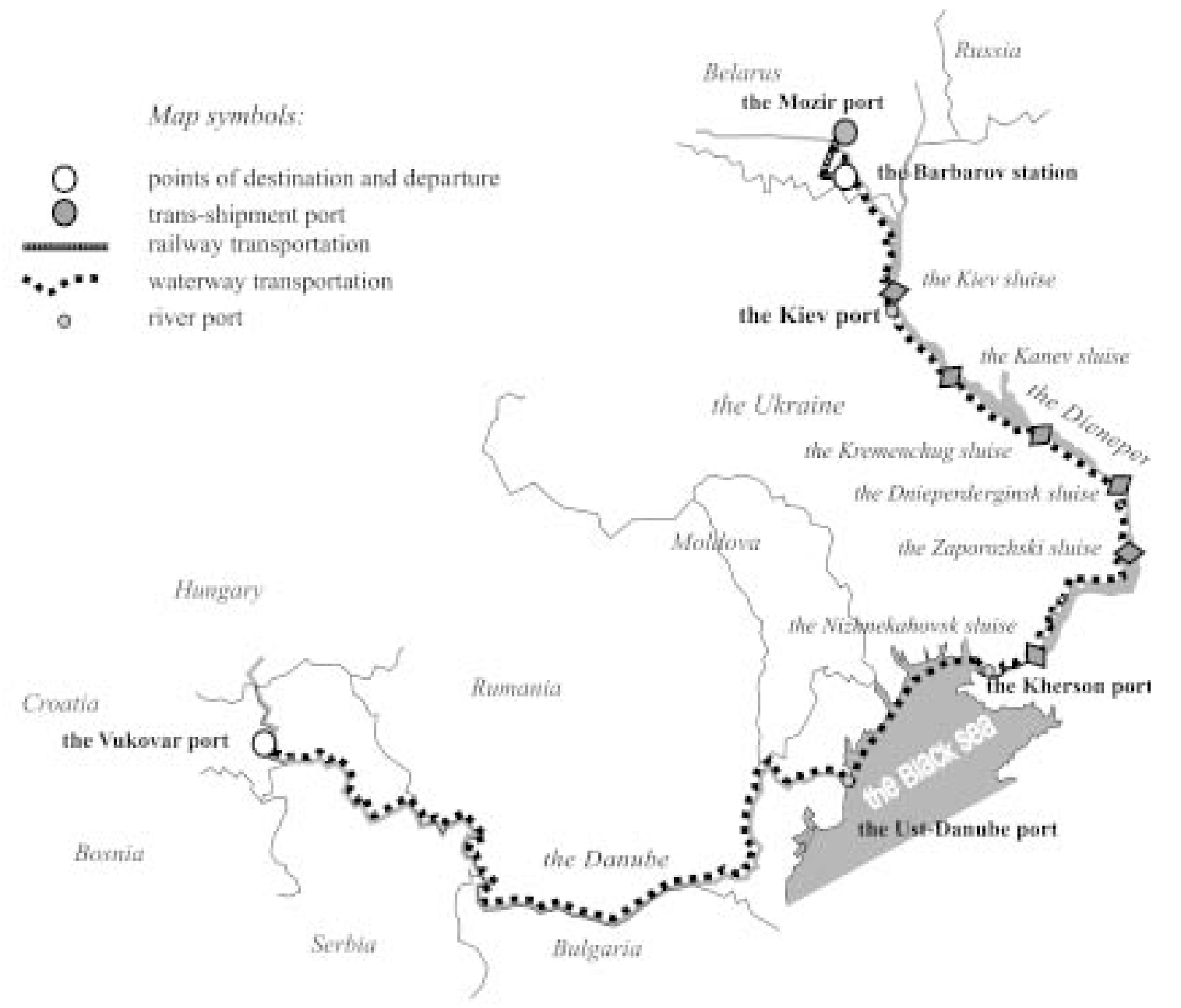

Fig 2. The scheme of waterway "- the Pripyat - the Dieneper - the Danube" 


$$
\begin{aligned}
& M 1 F_{8} \quad M 1_{3} \mid=M 1 F_{1}+\sum_{m=1}^{2} M 1_{m}+M 1 F_{3}+ \\
& \max \left(M 1 F_{4} ; M 1 F_{5}\right)+\max \left(M 1 F_{6} ; M 1 F_{7}\right)+ \\
& M 1 F_{8}+M 1_{3},
\end{aligned}
$$

where: $M 1 F_{1}$ is a module of vessel setting in the roadstead, the dissolution of the vessels group; $M 1_{1}$ is the supply of vessels according to the system services of fleet; $M 1_{2}$ is the expectation of cargo operations; $M 1 F_{3}$ is vessel setting to the loading wharf; $M 1 F_{4}$ are cargo operations; $M 1 F_{5}$ is documenting; $M 1 F_{6}$ is commercial and technical inspection of vessels; $M 1 F_{7}$ are customs cargo operations; $M 1 F_{8}$ is vessel putting off in the roadstead, the formation of vessel group; $M 1_{3}$ is the vessel putting off.

The servicing of the Belarusian fleet in the Kherson port (the Ukraine) (in the absence of cargo operations, for the purpose of getting the permission on sailing off (for the vessels of the "river-sea" class):

$$
\begin{aligned}
& M_{p U 3}^{2}=\left|\begin{array}{llllll}
M 1_{1} & M 1_{2} & M 1_{3} & M 1_{4} & M 1_{5} & M 1 F_{1}
\end{array}\right|= \\
& \sum_{m=1}^{5} M 1_{m}+M 1 F_{1},
\end{aligned}
$$

where: $M 1_{1}$ is waiting for the pilot; $M 1_{2}$ is the pilotage; $M 1_{3}$ is vessel setting in the roadstead; $M 1_{4}$ is the supply of vessels according to the system services of fleet (if it is necessary); $M 1_{5}$ is waiting for the permission to perform a sea passage; $M 1 F_{1}$ is vessel putting off.

On the grounds of the given material we can make a conclusion about the possibility of the adequate reflection of the enumerated modules of the $2^{\text {nd }}$ order by the composition union of the corresponding elementary modules, presenting the process of "multimodal cargo line" system functioning in interaction with each other.

For example, the multimodal cargo line on the transportation of potassium fertilizers (the Kaliy station, Belarus) to the port Belgrade (Republic of Serbia) with the sea lift from the port Mozir to the port Belgrade can be recorded thus:

$$
\begin{aligned}
& M_{M-B}^{1}=\mid M_{\text {ser } r t}^{2} M_{m o v ~ r t}^{2} M_{r e l}^{2} M_{m o v 1}^{2} M_{l o c 1}^{2} M_{m o v 2}^{2} \\
& M_{l o c 2}^{2} M_{m o v 3}^{2} M_{l o c 3}^{2} M_{m o v 4}^{2} M_{l o c 4}^{2} M_{m o v 5}^{2} M_{l o c 5}^{2} M_{m o v 6}^{2} \\
& M_{l o c 6}^{2} M_{m o v 7}^{2} M_{p U 3}^{2} M_{m o v 8}^{2} M_{l o c 3 \_1}^{2} M_{m o v 9}^{2} M_{l o c 3 \_2}^{2}
\end{aligned}
$$

$$
\begin{aligned}
& M_{m o v 10}^{2} M_{p D}^{2} M_{m o v 10}^{2} M_{l o c 3 \_2}^{2} M_{m o v 9}^{2} M_{l o c 3 \_1}^{2} M_{m o v}^{2} \\
& M_{m o v 7}^{2} M_{l o c 6}^{2} M_{m o v 6}^{2} M_{l o c 5}^{2} M_{m o v 5}^{2} M_{l o c 4}^{2} M_{m o v 4}^{2} M_{l o c 3}^{2} \\
& M_{m o v 3}^{2} M_{l o c 2}^{2} M_{m o v 2}^{2} M_{l o c 1}^{2} M_{m o v 1}^{2} \mid
\end{aligned}
$$

where: $M_{\text {ser } r t}^{2}$ is the module of the railway rolling stock servicing at the Kaliy station; $M_{m o v}^{2}$ is a motion from the Kaliy station to the Mozir port; $M_{r e l}^{2}$ is the transshipment of cargos in the port Mozir; $M_{\text {movl }}^{2}$ is a motion through the section "the port Mozir - the Kiev sluice"; $M_{\text {loc } 1}^{2}$ is sluicing in the Kiev sluice; $M_{\text {mov } 2}^{2}$ is a motion in the section "the Kiev sluice - the Kanev sluice"; $M_{l o c 2}^{2}$ is sluicing in the Kanev sluice; $M_{\text {mov } 3}^{2}$ is a motion through the section "the Kanev sluice - the Kremenchug sluice"; $M_{l o c 3}^{2}$ is sluicing in the Kremenchug sluice; $M_{\text {mov } 4}^{2}$ is a motion through the section "the Kremenchug sluice - the Dnieperderginsk sluice"; $M_{\text {loc } 4}^{2}$ is sluicing in the Dnieperderginsk sluice; $M_{\text {mov } 5}^{2}$ is a motion through the section "the Dnieperderginsk sluice - the Zaporozh'ye sluice"; $M_{l o c 5}^{2}$ is sluicing in the Zaporozh'ye sluice; $M_{\text {mov }}^{2}$ is a motion through the section "the Zaporozh'ye sluice - the Nizhnekahovsk sluice"; $M_{l o c 6}^{2}$ is sluicing in the Nizhnekahovsk sluice; $M_{\text {mov } 7}^{2}$ is a motion through the section "the Nizhnekahovsk sluice - the Kherson port"; $M_{p U 3}^{2}$ is a calling of the vessel at the Kherson port to wait for the permission of the sea passage; $M_{\text {mov }}^{2}$ is a motion through the section "the Kherson port - the Iron Gates II sluice"; $M_{\text {loc } 3 \text { _1 }}^{2}$ is sluicing; $M_{\text {mov } 9}^{2}$ is a motion through the section "the Iron Gates II sluice - the Iron Gates I sluice"; $M_{l o c 322}^{2}$ is sluicing; $M_{\text {mov10 }}^{2}$ is a motion through the section "the Iron Gates I sluice - the Belgrade port"; $M_{p D}^{2}$ is the fleet servicing in the Vukovar port.

The complex system being researched (multimodal cargo lines group) in accordance with the modular principle of the model formation is specified as the totality of the $1^{\text {st }}$ order modules, the interaction of which is effected by means of the transition matrices determined by the system structure and the priorities of the requests servicing. Thus, for example, with the simultaneous arrival of not self-propelled vessels group, the motion of which is organized as a part of one multimodal cargo line, and of the combined navigation motorship (along another cargo rout) into one and the same port, the latter possesses more significant priority.

A common form of the algorithm for choosing the optimum scheme of export-import cargos of Belarus multimodal transportation organization on the basis of simulation modeling is represented in Fig 3. 


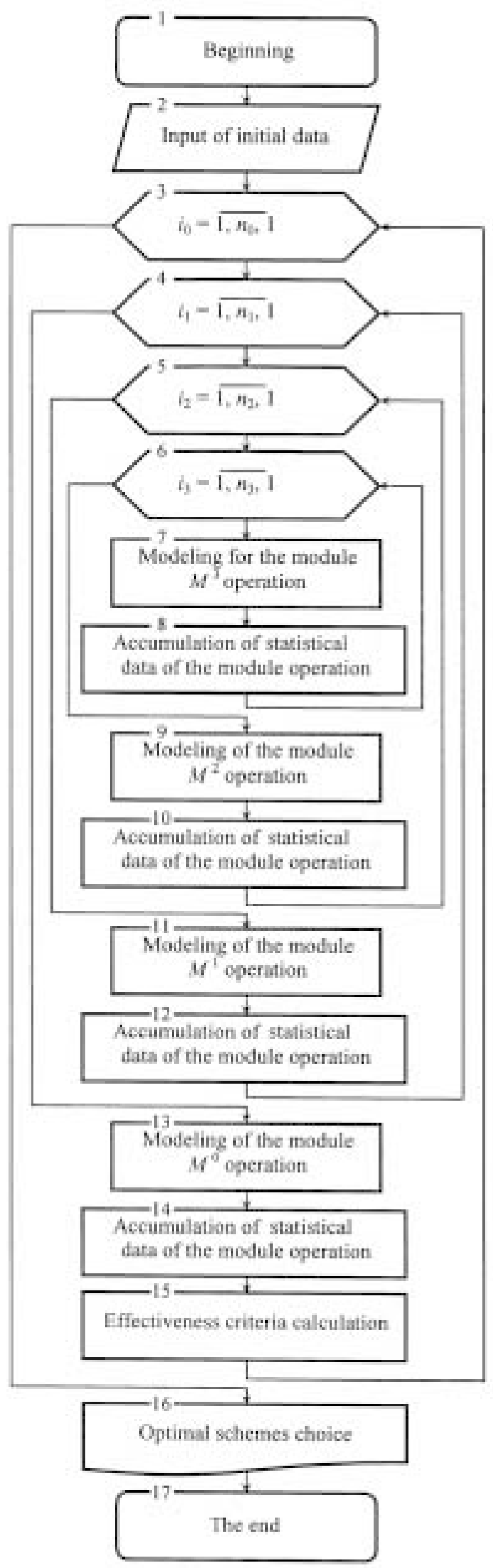

Fig 3. Algorithm for choosing the optimum scheme of export-import cargos of Belarus multimodal transportation organization
Some comments for the algorithm which is presented in Fig 3:

3: Cycle according to parameter $i_{0}$ is changing in the range from 1 to $n_{0}$ (quantity of multimodal lines grouping versions) with step 1

4: Cycle according to parameter $i_{1}$ is changing in the range from 1 to $n_{1}$ (quantity of multimodal cargo lines in the group $i_{0}$ ) with step 1

5: Cycle from parameter $i_{2}$ is changing in the range from 1 to $n_{2}$ (quantity of operations on the cargo delivery in the multimodal cargo line $i_{1}$ ) with step 1

6: Cycle from parameter $i_{3}$ is changing in the range from 1 to $n_{3}$ (quantity of elementary modules in the operation on the cargo delivery $i_{2}$ ) with step 1

7: Block of elementary operations modeling

8: Accumulation of statistical data about the fulfillment of elementary operations

9: Block of modeling operations, constituting the multimodal cargo line

10: Accumulation of statistical data about the fulfillment of operations on the cargo delivery

11: Block of the multimodal cargo line functioning modeling

12: Accumulation of statistical data about the multimodal cargo lines functioning

13: Block of modeling multimodal cargo lines group functioning, the algorithm

14: Accumulation of statistical data about the multimodal cargo lines group functioning

15: Calculation of the effectiveness criteria of the organization of the transportation in the cargo lines group

Each module, as it was said above, is characterized by the parameters (formula 1) identifying module functioning according to different variants of the transport process organization. Therefore, in order to describe adequately the process of functioning of the model of the system being researched taking into account the specific character of each module which is its part and technology of each separate request servicing, it is necessary to specify not only the technological interactions of modules, but also the parameters of their operation modeling. Such as: the form of the law of the request servicing time distribution, the parameters of this law, the form of the task solved by module etc.

The form and the parameters of the distribution law can be obtained on the basis of statistical data processing, and in the case of this information absence, it is possible to use the wide experience of similar problems solution by scientists, who research the questions of different modes of transport exploitation. 
Algorithms of random variables generation with the specified characteristics of the distribution laws were developed and have been successfully used since the beginning of the wide use of a simulation modeling method to research complex dynamic systems and they were presented in the works of G. Benks, N. P. Buslenko, V. I. Savin, S. M. Pianih, etc. Naturally, with the program realizations of simulation models it is necessary to introduce changes into standard algorithms. These changes are directed to the specific character of the task stated by a researcher and are conditioned by the use of different effectiveness criteria of optimum versions.

\section{Conclusion}

With the help of the simulation model of multimodal cargo line formed according to the above principles it is possible to research different aspects of transport objects exploitation, questions of coordination and interaction of the modes of transport and other questions, for example, questions which are connected with the specific character of multimodal transportation organization [5]. In this case, depending on the required accuracy of calculations one can select a different degree of model detailing. In the described example, when the estimation of the effectiveness of a number of export and import cargos transportation organization system is the result of modeling, it is quite enough to represent the researched system in the form of the $1^{\text {st }}$, the $2^{\text {nd }}$ and the $3^{\text {rd }}$ of orders modules totalities. For further detailing of the model, the modules of the $3^{\text {rd }}$ order (technological operations) can be in their turn represented as the totality of the modules of the $4^{\text {th }}$, the $5^{\text {th }}$ and higher orders, however, in this case one should take into consideration that the expenditures of processing time for this system modeling will considerably grow.

\section{References}

1. Kazakov, N. N. Research of the efficiency of development of freight traffics by river transport of Byelorussia. In: The international collection of proceedings "Complex operation of types of transport” (Международный сборник научных трудов „Комплексная эксплуатация видов транспорта“). Gomel: BelSUT, 2004, p. 71-77 (in Russian).

2. Malyshkin, A. G. The organization and scheduling of river fleet (Организация и планирование работы речного флота). Moscow: Transport, 1985. 215 p. (in Russian).

3. Banks, J.; Carson, J.; Nelson, B. Discrete-event system simulation. Second edition, Prentice-hall, Englewood Cliffs, New Jersey, 1996. 594 p.
4. Buslenko, N. P. Mathematical modelling of productions on digital computers (Математическое моделирование производственных процессов на цифровых вычислительных машинах). Moscow: Science, 1964. 362 p. (in Russian).

5. Nikiforov, V. S. Multimodal transportations and transport logistics (Мультимодальные перевозки и транспортная логистика). Novosibirsk: NSAWT, 1999. 104 p. (in Russian). 